\title{
BMJ Open Chronic heart failure patients' experiences of German healthcare services: a protocol for a scoping review
}

\author{
Mirjam Dieckelmann, ${ }^{1}$ Felix Reinhardt, ${ }^{1}$ Klaus Jeitler, ${ }^{2}$ Thomas Semlitsch, ${ }^{2}$ \\ Jasper Plath, ${ }^{1}$ Ferdinand M Gerlach, ${ }^{1}$ Andrea Siebenhofer, ${ }^{1,2}$ Juliana J Petersen ${ }^{1}$
}

To cite: Dieckelmann M, Reinhardt F, Jeitler K, et al. Chronic heart failure patients' experiences of German healthcare services: a protocol for a scoping review. BMJ Open 2019;9:e025685. doi:10.1136/ bmjopen-2018-025685

- Prepublication history and additional material for this paper are available online. To view these files, please visit the journal online (http://dx.doi. org/10.1136/bmjopen-2018025685).

Received 26 July 2018

Revised 26 November 2018

Accepted 18 December 2018

Check for updates

(C) Author(s) (or their employer(s)) 2019. Re-use permitted under CC BY-NC. No commercial re-use. See rights and permissions. Published by BMJ.

${ }^{1}$ Institute of General Practice, Goethe University Frankfurt,

Frankfurt, Germany

${ }^{2}$ Institute of General Practice and Evidence-based Health Services Research, Medical University of Graz, Graz, Austria

\section{Correspondence to}

Mirjam Dieckelmann;

Dieckelmann@

allgemeinmedizin.uni-frankfurt. de

\section{ABSTRACT}

Introduction Chronic heart failure (CHF) is a heterogeneous condition requiring complex treatment from diverse healthcare services. An increasingly holistic understanding of healthcare has resulted in contextual factors such as perceived quality of care, as well as patients' acceptance, preferences and subjective expectations of health services, all gaining in importance. How patients with CHF experience the use of healthcare services has not been studied within the scope of a systematic review in a German healthcare context. The aim of this scoping review is therefore to review systematically the experiences of patients affected by CHF with healthcare services in Germany in the literature and to map the research foci. Further objectives are to identify gaps in evidence, develop further research questions and to inform decision makers concerned with improving healthcare of patients living with CHF.

Methods and analysis This scoping review will be based on a broad search strategy involving systematic and comprehensive electronic database searches in MEDLINE, EMBASE, PsycINFO, PSYNDEX, CINAHL and Cochrane's Database of Systematic Reviews, grey literature searches, as well as hand searches through reference lists and non-indexed key journals. The methodological procedure will be based on an established six-stage framework for conducting scoping reviews that includes two independent reviewers. Data will be systematically extracted, qualitatively and quantitatively analysed and summarised both narratively and visually. To ensure the research questions and extracted information are meaningful, a patient representative will be involved.

Ethics and dissemination Ethical approval will not be required to conduct this review. Results will be disseminated through a clearly illustrated report that will be part of a wider research project. Furthermore, it is intended that the review's findings should be made available to relevant stakeholders through conference presentations and publication in peer-reviewed journals (knowledge transfer). Protocol registration in PROSPERO is not applicable for scoping reviews.

\section{INTRODUCTION}

Heart failure is a major chronic condition affecting a growing number of people in Germany, most of whom are older adults. ${ }^{1}$ A diagnosis of heart failure (ICD-I50) is the

\section{Strengths and limitations of this study}

- This scoping review will be the first to systematically map the past 10 years of research activity surrounding chronic heart failure patients' experiences of using healthcare services in Germany and to genuinely explore the consumer perspective.

- The research will be limited to Germany but will allow the replication of findings for other healthcare systems with their specific geographical and institutional healthcare features.

- All healthcare settings and study designs will be relevant for inclusion in this review to provide an overview of the entire range of German health services.

- A patient representative was involved in refining the research questions and defining the outcome variables to be extracted.

- Since patient experience is a broad concept, we expect study heterogeneity to lead to potential difficulties in summarising extracted data.

second most common cause of death among women and is one of the top four causes of death among men in Germany. ${ }^{2}$ Due to the bidirectional interplay between somatic and psychosocial factors, patients with chronic heart failure (CHF) experience both reduced survival and quality of life. ${ }^{3}$ Evidence suggests that with increasing severity of clinical symptoms, quality of life decreases disproportionately in hospitalised ${ }^{4}$ and primary care patients. $^{5}$

In Germany, approximately $€ 5.3$ billion were spent on treating heart failure in $2015 .^{6}$ Having doubled over the past two decades, it is the most common primary reason for hospital admission in Germany ${ }^{2}$ and is a major cause of in-hospital mortality. ${ }^{7}$

Both the overall economic burden of the disease and the individual physical, mental and psychosocial impact on CHF patients' lives mean health services must coordinate their services well if patients' complex healthcare needs are to be managed effectively and efficiently. However, repeated hospital 
admissions following acute decompensation are characteristic of patients with CHF and make it difficult for inpatient and outpatient care sectors to coordinate their activities. $^{89}$

The management of CHF should be minimally disruptive and involve multidisciplinary healthcare services, both in formal healthcare facilities and the patient's environment. ${ }^{10}$ The European Society of Cardiology has identified the following as areas in which healthcare provision can potentially be improved: discharge planning, lifestyle advice, exercise training, follow-up and monitoring, palliative and end-of-life-care as well as self-care in elderly, frail or cognitively impaired patients with CHF. ${ }^{10}$

In modern healthcare systems, patients with CHF find themselves managing their condition both at home and, when seeking help from the confusing range of healthcare providers and their various services, externally. The burden of treatment may be perceived as challenging as a result of the need to adhere to complex pharmaceutical therapies, manage comorbidities that interfere with CHF, physically and psychologically cope with symptoms that may severely interfere with everyday life activities, continuously monitor one's own health status, operate technical devices, understand complex disease mechanisms and interact with different health professionals. ${ }^{11}$

To help patients cope with such immense workloads, a disease management programme for patients with $\mathrm{CHF}$ is scheduled for implementation in Germany in 2018. ${ }^{12}$ Against this background, this review's findings may help policy makers, healthcare providers and decision makers concerned with intersectoral or intrasectoral management of care to gain a comprehensive overview of what is known about the individual healthcare needs of patients with $\mathrm{CHF}$ and their experiences and satisfaction with the healthcare they receive in Germany.

The literature on measuring quality of care suggests that no consensus has yet been reached on which patient-relevant aspects should be included in the development of quality indicators. ${ }^{13}$ Identification of the scope of evidence will therefore help to understand what is most important to the patient receiving healthcare. Healthcare organisations concerned with improving the quality of services in CHF care may use this knowledge to define what constitutes a positive patient experience and infer factors that may improve healthcare provision for this target group.

Furthermore, this knowledge may lay the groundwork for the development of a disease-specific assessment tool to measure how CHF patients' experience their treatment in Germany. Different approaches for measuring patients' experiences have been reviewed in literature, suggesting that there is no gold standard for such an assessment. ${ }^{14}$ Hence, this evidence map seeks to review a wide array of methodological study designs that report on patients' experiences.

Emphasis on a holistic approach for understanding healthcare makes it particularly important to review the effectiveness of medical interventions and to take into account contextual factors such as patients' lived experiences, as well as their acceptance and expectations of healthcare services. A meta-synthesis of qualitative reviews has found that patients' experiences along with rational choices establish how patients make decisions relating to self-care and help-seeking behaviour in the management of their complex chronic conditions. ${ }^{15}$ For CHF, prior experiences can be expected to influence decisions concerning management of the disease, implementation of a healthy lifestyle, effective cooperation with health professionals and adherence to treatment regimens, all of which ultimately affect mortality, quality of life and other relevant outcomes. ${ }^{16}$

However, little is known about the subjective experiences of patients with CHF that are specifically confronted with the German healthcare infrastructure, and seeking and receiving healthcare services. A preliminary search for existing and registered reviews revealed no such evidence. Although a comprehensive scoping study exploring the literature on patients' experiences is underway, it focuses on integrated care settings rather than a country-specific healthcare landscape. ${ }^{17}$ To the best of our knowledge, this is the first scoping review conducted to ask what existing literature can tell us about the experiences of patients with CHF with the entire range of German health services in both formal and informal care settings.

\section{METHODS AND ANALYSIS}

Since standard reporting guidelines such as Preferred Reporting Items for Systematic Reviews and Meta-Analyses Extension for Scoping Reviews (PRISMA-ScR) are currently being developed, ${ }^{18}$ the methodological procedure followed in this scoping review is based on the theoretical framework of Arksey and O'Malley, ${ }^{19}$ and the complementary recommendations proposed by Levac and colleagues. ${ }^{20}$ This six-stage approach also complies with recommendations on conducting scoping reviews published by The Joanna Briggs Institute. ${ }^{21}$ The procedure consists of the following six steps: (1) identifying the research question; (2) identifying relevant studies; (3) study selection; (4) charting the data; (5) collating, summarising and reporting the results and (6) consultation.

\section{Identifying the research question}

The aim of this review is to provide an accessible overview of what is known about CHF patients experiences of using healthcare services in Germany.

Following on from the above objective, the main research questions is:

1. What is the extent and range of available evidence addressing CHF patients' experiences of using German healthcare services?

In order to understand patients' journeys through the German healthcare system and to shed light on their subjective experiences on these journeys, secondary research questions are: 
2. What healthcare services and settings, what geographical contexts and what dimensions of patient experience are covered?

3. What methodological designs are used to assess patients' experiences?

To examine the full range of patients' experiences, we have defined the multidimensional concept of patient experience rather broadly. To the best of our knowledge, a uniform definition of patients' experiences does not yet exist in the literature. Based on narrative synthesis reviews, we have therefore developed the following working definition ${ }^{22} 23$ : patient experience consists of the multitudinous interactions originating at the interface between disease specific, subjective healthcare needs and the healthcare services used by patients. They encompass various dimensions-whether they be relational, organisational or functional in nature-either obtained from the patients themselves, or otherwise accurately reflecting the patient's views.

We applied the (population - concept context (PCC)-mnemonic introduced by The Joanna Briggs Institute ${ }^{21}$ to develop the research question. For this scoping review, population $(\mathrm{P})$ is defined as adults living with CHF. The concept of interest (C) constitutes the experiences of patients managing the complex condition of CHF that result from their disease-specific healthcare needs on the one hand and the healthcare they receive on the other. The context $(\mathrm{C})$ of the research question includes all formal and informal healthcare services provided in all healthcare settings in Germany.

\section{Identifying relevant studies}

The search strategy will be broad and will aim to cover literature, including grey literature, from various academic disciplines. This is necessary since investigating patients' experiences in different German healthcare settings may involve research fields as diverse as psychology, medical sciences, healthcare management, nursing, rehabilitation and e-health.

Consequently, we will conduct systematic literature searches in the following six databases: CINAHL, MEDLINE, EMBASE (all via Ovid), PsycINFO, PSYNDEX (both via EBSCO) and the Cochrane Database of Systematic Reviews via Wiley.

Additionally, a non-systematic search of grey literature will be undertaken using platforms such as Google Scholar, Open Grey and ProQuest, since research articles that have not been peer-reviewed will also be relevant. The same holds true for experts and organisations involved in the field of patient-experienced healthcare, whom we also intend to contact.

Finally, we will hand search the reference lists of included studies and search the indexes of key journals.

A first step in developing the systematic search strategy was to use the PCC-mnemonic to brainstorm for search terms that well describe the population, the concept under investigation and the context of the research question. Additional search terms were extracted from scans of titles and abstracts in an initial non-exhaustive search.

Search strategies for all databases and publication dates of coverage are provided in an online supplementary file in the appendix. They comprise three search blocks that reflect the PCC-scheme. In addition, searches will be limited to publications in German and English that have been published within the last 10 years (2008 to present). Preliminary searches across all databases were conducted in April 2018. Final searches are scheduled to be finished by the end of 2018 (database searches and grey literature searches). Once the search strategy has been refined through the incorporation of feedback from our research team members and run electronically, identified articles will be exported and converted into the standard file format Research Information Systems (RIS). Subsequently, we will merge all identified studies into Covidence software ${ }^{24}$ for deduplication and the selection of further studies.

\section{Study selection}

Two independent reviewers will screen titles, abstracts and full texts of the articles. A majority vote involving a third reviewer will resolve any disagreements on which studies to include on all levels of the search. The PRISMA flow diagram will be used to give an overview of the number of studies throughout the study selection process. ${ }^{25}$

All research articles that address the experiences of using healthcare services of adults receiving treatment in Germany after a diagnosis of CHF (as defined in the individual studies) will be eligible for this review.

All clinical forms of CHF are relevant for inclusion, regardless of aetiology or classification of severity. Studies dealing with patients with multiple chronic conditions or comorbidities will also be eligible for inclusion if patients' experiences specifically relate to CHF.

For the review, patient experience will be defined broadly and include neighbouring concepts such as patients' preferences, needs, values, satisfaction, burden of treatment and expectations of healthcare services. Study designs assessing patients' experiences indirectly that is, expert opinion, routine data that was not obtained from patients directly, or came from health professionals, will not be eligible for inclusion. On the other hand, since informal caregivers play a significant role in shaping patients' experiences of healthcare services, studies that report patient experiences from the perspective of a patient's close social network will be eligible for inclusion-especially towards end of life and when patients are unable to self-report their experiences. ${ }^{26}$

All healthcare settings will be considered relevant for this review, including traditional settings of inpatient and outpatient care, as well as other health services that patients use to manage their CHF. As we are aware that health systems differ substantially between countries, we intend to classify healthcare services based on Busse and Blümel's categorisation of provision of services, as 
reported in a series published by the European Observatory on Health Systems and Policies. ${ }^{27}$ In this way, it will be possible to draw conclusions from the review's results for healthcare systems in different countries.

We will exclude publications that involve data from multiple countries unless the study design allows the separate analysis of data for Germany. Studies investigating patients' experiences of using non-medical and social services, as well as non-research publications such as online ratings and consumer blogs, will not be considered eligible for this review. Furthermore, when patients' experiences focus on perceived burden of disease, rather than directly relating to the use of healthcare services, they will also be excluded.

\section{Charting the data}

A data-charting form will be used for all eligible full texts. The development of the data-charting forms will involve all multidisciplinary research team members. A patient representative will also be involved to discuss the importance of outcome variables from a healthcare user perspective. Data extraction will be piloted using two relevant studies to ensure applicability and will be updated if necessary. In the next step, data will be extracted based on the three categories listed in table 1.

First, study characteristics will be extracted, including data on the first author's name, year and type of publication, academic discipline, sample size, study objective as well as the main conclusion.

Second, key findings will be extracted, depending on their relevance to the research questions. The findings will be described in terms of whether they can be categorised as positive, negative or both, the geographical setting (ie, federal state, area code, urban vs rural area), the institutional setting or healthcare service used (ie, inpatient hospital care, informal home care, rehabilitation services) and the methodology used to assess patients' experiences.

Third, population characteristics will be charted. These will include data on age, sex, ethnicity, insurance and relationship status, severity of symptoms/NYHA, comorbidities, type of CHF as well as therapeutic characteristics (ie, implanted device therapy, pharmacological therapy).

\section{Collating, summarising and reporting the results}

First of all, information on study and population characteristics from the data-charting form will be summarised using basic descriptive statistics and descriptive narration. Subsequently, key findings including information on study methodology, categories of patient experience, authors' conclusions, types of healthcare service, the institutional setting under investigation as well as the geographical setting of the study will be tabulated and summarised narratively.

To visually depict the quantity of research across various study designs and healthcare settings, an evidence map will be created in a second step. If appropriate, the evidence map will be a bubble diagram that includes the following
Table 1 Data charting form used to extract information to answer the scoping review's questions

\begin{tabular}{|c|c|}
\hline Domain & Extracted Information \\
\hline \multirow[t]{7}{*}{ Study characteristics } & First author \\
\hline & Year of publication \\
\hline & Type of publication \\
\hline & Academic discipline \\
\hline & Sample size \\
\hline & Study objective \\
\hline & Authors' main conclusion \\
\hline \multirow[t]{5}{*}{ Key findings } & $\begin{array}{l}\text { Thematic dimension of patient } \\
\text { experience }\end{array}$ \\
\hline & Experience: positive/negative/both \\
\hline & $\begin{array}{l}\text { Geographical setting (ie, federal } \\
\text { state, area code, urban vs rural } \\
\text { area) }\end{array}$ \\
\hline & $\begin{array}{l}\text { Institutional setting or healthcare } \\
\text { service used (ie, inpatient hospital } \\
\text { care, informal home care, } \\
\text { rehabilitation services) }\end{array}$ \\
\hline & $\begin{array}{l}\text { Method used to assess patients' } \\
\text { experiences }\end{array}$ \\
\hline \multirow{9}{*}{$\begin{array}{l}\text { Population } \\
\text { characteristics }\end{array}$} & Age \\
\hline & Sex \\
\hline & Ethnicity \\
\hline & Insurance status \\
\hline & Relationship status \\
\hline & Severity of symptoms (NYHA) \\
\hline & Comorbidities \\
\hline & $\begin{array}{l}\text { Type of CHF (in terms of } \\
\text { localisation, aetiology or } \\
\text { pathophysiology) }\end{array}$ \\
\hline & $\begin{array}{l}\text { Therapeutic characteristics } \\
\text { (ie, implanted device therapy, } \\
\text { pharmacological therapy) }\end{array}$ \\
\hline
\end{tabular}

CHF, chronic heart failure; NYHA, New York Heart Association Functional Classification.

reported information: type of studies, number of eligible studies, geographical setting, healthcare service and institutional setting.

Both the visual and narrative presentation of results will provide information on the breadth of knowledge available on the experiences of patients with CHF with German healthcare services. A basic thematic analysis of what was reported in eligible studies will be undertaken by summarising key findings, while the creation of a bubble plot will highlight gaps in the evidence. The kinds of study designs that are relevant to the field will be listed on the bubble plot's x-axis, and the types of healthcare service relevant to patients with CHF in Germany on the 
y-axis. These specifications will be refined by research staff in consultation with a patient representative.

\section{Consultation}

The aim of the review is to provide an accessible overview of CHF patients' experiences of using different kinds of healthcare services in Germany, as portrayed in the literature.

In line with this objective, we will make use of end-ofproject knowledge translation by incorporating stakeholders with backgrounds in healthcare research, clinical practice, nursing and the organisation of patient support groups. These stakeholders will form an advisory board that is part of a wider research project, whereby board meetings will serve as a platform to elucidate which key messages most appropriately address end-users' information needs.

\section{Patient and public involvement}

Investigating patients' experiences requires consultation with patients to ensure extraction of both appropriate and meaningful outcome variables. A patient representative was therefore involved in refining the research questions and developing a data-charting form, and the matrix for a bubble plot. For this purpose, we conducted semistructured telephone interviews with the patient representative, or sent the representative questionnaires with open questions via email. Apart from the specification of outcome variables and research questions, patients will not be involved in the overall design of the study, conducting review processes, data extraction and the representation of results.

\section{ETHICS AND DISSEMINATION}

Using scoping review methodology, we aim to systematically and comprehensively map available literature on CHF patients' experiences of using healthcare services in Germany. The rationale for choosing the scoping review methodology and deciding against undertaking a critical appraisal of included studies is twofold. First, clustering research patterns and gaps in patient-centred CHF health services research will provide valuable information for the development of further research questions for primary research, or full systematic research syntheses, regardless of whether they take a qualitative, quantitative or mixed-methods approach. Second, decision makers and practitioners in healthcare will be provided with a comprehensive overview of the extent and nature of the past decade's research activity that relates to their specific healthcare context.

This scoping review will be conducted by a multidisciplinary research team that includes members with diverse backgrounds such as health services research, psychology, healthcare management and public health. This scoping review will be embedded within a wider research project that aims to develop a clearly illustrated report on current structures of CHF management within the German healthcare system. As such, its results will be disseminated in this report and presented to relevant stakeholders at the advisory board meeting. Moreover, we intend to present findings from this scoping review at research conferences and to publish them in a peer-reviewed journal.

Acknowledgements We would like to thank Phillip Elliott for proof-reading the manuscript. Furthermore, we would like to gratefully acknowledge the valuable advice provided by the patient representative, Helmuth Bundschuh.

Contributors JJP, AS, FMG: developed the concept and acquired funding for this review. MD, KJ, TS, FR: developed the search strategy. MD: drafted the first version of the manuscript. JJP, KJ, TS, FR, JP: finalised this manuscript.

Funding This work was supported by the Robert Bosch Foundation (Robert Bosch Stiftung).

Competing interests JJP is a co-investigator in the PANORA study (Prevalence of anticyclic citrullinated peptide (CCP) positivity in patients with new non-specific onset of musculoskeletal symptoms, possibly related to early rheumatoid arthritis in general practices in Germany), which is being conducted by the Fraunhofer Institute and financed by Bristol-Meyer Squibb. She is employed by the Institute of General Practice of Goethe University Frankfurt and has never personally received financial remuneration from a pharmaceutical company.

Patient consent for publication Not required.

Ethics approval This study will not require ethical approval.

Provenance and peer review Not commissioned; externally peer reviewed.

Open access This is an open access article distributed in accordance with the Creative Commons Attribution Non Commercial (CC BY-NC 4.0) license, which permits others to distribute, remix, adapt, build upon this work non-commercially, and license their derivative works on different terms, provided the original work is properly cited, appropriate credit is given, any changes made indicated, and the use is non-commercial. See: http://creativecommons.org/licenses/by-nc/4.0/.

\section{REFERENCES}

1. Ohlmeier C, Mikolajczyk R, Frick J, et al. Incidence, prevalence and 1-year all-cause mortality of heart failure in Germany: a study based on electronic healthcare data of more than six million persons. Clin Res Cardiol 2015;104:688-96.

2. Neumann T, Biermann J, Erbel R, et al. Heart failure: the commonest reason for hospital admission in Germany: medical and economic perspectives. Dtsch Arztebl Int 2009;106:269-75.

3. Hermann-Lingen C. Herzinsuffizienz und Herztransplantation. Cardiac insufficiency and heart transplantation. In: Herrmann-Lingen C, Boll-Klatt A, Psychokardiologie: ein praxisleitfaden für ärzte und psychologen [psycho-cardiology: a best practice guide for doctors and psychologists. 2nd edn. Köln: Dt. Ärzte-Verl, 2014:184-200.

4. Scherer M, Stanske B, Wetzel D, et al. Die krankheitsspezifische lebensqualität von hausärztlichen patienten mit herzinsuffizienz [disease-specific quality of life in ambulatory patients with cardiac insufficiency] zeitschrift für ärztliche fortbildung und qualität im gesundheitswesen - German. J for Quality in Health Care 2007;101:185-90.

5. Juenger J, Schellberg D, Kraemer S, et al. Health related quality of life in patients with congestive heart failure: comparison with other chronic diseases and relation to functional variables. Heart 2002;87:235-41.

6. Statistisches Bundesamt Krankheitskostenrechnung. Krankheitskosten in mio. $€$ für deutschland: gliederungsmerkmale: Jahr, Alter Geschlecht, ICD10 (I50 Herzinsuffizienz) [German medical expenses in $€$ millionsaccording to year, age, and sex, ICD10]. http:// www.gbe-bund.de/ (Accessed 13 Nov 2018).

7. Christ M, Störk S, Dörr M, et al.Heart failure epidemiology 20002013: insights from the german federal health monitoring system. Eur $J$ Heart Fail 2016;18:1009-18.

8. Arzneimittelkommission der Deutschen Apotheker, Arzneimittelkommission der deutschen Ärzteschaft, Deutsche Arbeitsgemeinschaft Selbsthilfegruppen e. V., et al. NVL Chronische Herzinsuffizienz - Langfassung, 2. Auflage [German Clinical Practice Guideline for Chronic Heart Failure]: Bundesärztekammer (BÄK); Kassenärztliche Bundesvereinigung (KBV); Arbeitsgemeinschaft der Wissenschaftlichen Medizinischen Fachgesellschaften (AWMF). 2017. 
9. Edelmann F, Knosalla C, Mörike K, et al. Chronic Heart Failure. Dtsch Arztebl Int 2018;115:124-30.

10. Ponikowski P, Voors AA, Anker SD, et al. 2016 ESC Guidelines for the diagnosis and treatment of acute and chronic heart failure: The Task Force for the diagnosis and treatment of acute and chronic heart failure of the European Society of Cardiology (ESC)Developed with the special contribution of the Heart Failure Association (HFA) of the ESC. Eur Heart J 2016;37:2129-200.

11. Park CL, Malone MR, Suresh DP, et al. Coping, meaning in life, and quality of life in congestive heart failure patients. Qual Life Res 2008;17:21-6.

12. Gemeinsamer Bundesausschuss Stabsabteilung Öffentlichkeitsarbeit. Strukturierte behandlung der herzinsuffizienz künftig in eigenständigem disease-management-programm [disease management program for cardiac insufficiency to be launched in the future]. $2018 \mathrm{https}: / / w w w . g-b a . d e / i n s t i t u t i o n / p r e s s e /$ pressemitteilungen/746/ (Accessed 13 Nov 2018).

13. Pohontsch NJ, Herzberg $\mathrm{H}$, Joos $\mathrm{S}$, et al. The professional perspective on patient involvement in the development of quality indicators: a qualitative analysis using the example of chronic heart failure in the German health care setting. Patient Prefer Adherence 2015;9:151-9.

14. de SD. Measuring patient experience: Evidence Scan. 2013.

15. May CR, Cummings A, Myall M, et al. Experiences of long-term life-limiting conditions among patients and carers: what can we learn from a meta-review of systematic reviews of qualitative studies of chronic heart failure, chronic obstructive pulmonary disease and chronic kidney disease? BMJ Open 2016;6:e011694.

16. Moser DK, Watkins JF. Conceptualizing self-care in heart failure: a life course model of patient characteristics. J Cardiovasc Nurs 2008;23:205-18.
17. Youssef AT, Constantino R, Chaudhary ZK, et al. Mapping evidence of patients' experiences in integrated care settings: a protocol for a scoping review. BMJ Open 2017;7:e018311.

18. The EQUATOR Network. Reporting guidelines under development

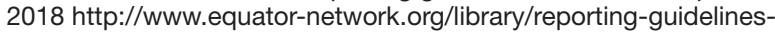
under-development/\#55 (Accessed 12 June 2018).

19. Arksey H, O'Malley L. Scoping studies: towards a methodological framework. Int J Soc Res Methodol 2005;8:19-32.

20. Levac D, Colquhoun H, O'Brien KK. Scoping studies: advancing the methodology. Implement Sci 2010;5:69.

21. The Joanna Briggs Institute. Joanna briggs institute reviewers' manual. 2015.

22. National Clinical Guideline Centre at The Royal College of Physicians. Patient experience in adult NHS services: improving the experience of care for people using adult NHS services: Patient experience in generic terms. Clinical Guidance. Methods, evidence and recommendations. 2012. https://www.nice.org.uk/guidance/ cg138 (Accessed 17 Nov 2018).

23. Wolf JA, Niederhauser $\mathrm{V}$, Marshburn D, et al. Defining patient experience. Patient Experience Journal 2014;1.

24. Veritas Health Innovation. Covidence systematic review software. Melbourne, Australia: Veritas Health Innovation.

25. Liberati A, Altman DG, Tetzlaff $\mathrm{J}$, et al. The PRISMA statement for reporting systematic reviews and meta-analyses of studies that evaluate healthcare interventions: explanation and elaboration. $B M J$ 2009;339:b2700.

26. Stocker R, Close $\mathrm{H}$, Hancock $\mathrm{H}$, et al. Should heart failure be regarded as a terminal illness requiring palliative care? A study of heart failure patients', carers' and clinicians' understanding of heart failure prognosis and its management. BMJ Support Palliat Care 2017:7:464-9.

27. Busse R, Blümel M. Germany: health system review. Health Syst Transit 2014;16:179-236. 